\title{
Self-renewing macrophages
}

The ability to self-renew is an essential feature of progenitor cells and is usually lost on differentiation. However, Aziz et al. now report that the deletion of two transcription factors linked to monocyte differentiation could confer limitless self-renewal capacity to mature differentiated macrophages. Surprisingly, despite their extended proliferative capacity, these macrophages were not tumorigenic and they could function in normal immune responses, making them attractive candidates for monocyte-based cellular therapies.

Myeloid progenitor cells proliferate to form colonies when cultured in the presence of macrophage colony-stimulating factor (M-CSF; also knwn as CSF1), whereas differentiated blood monocytes and

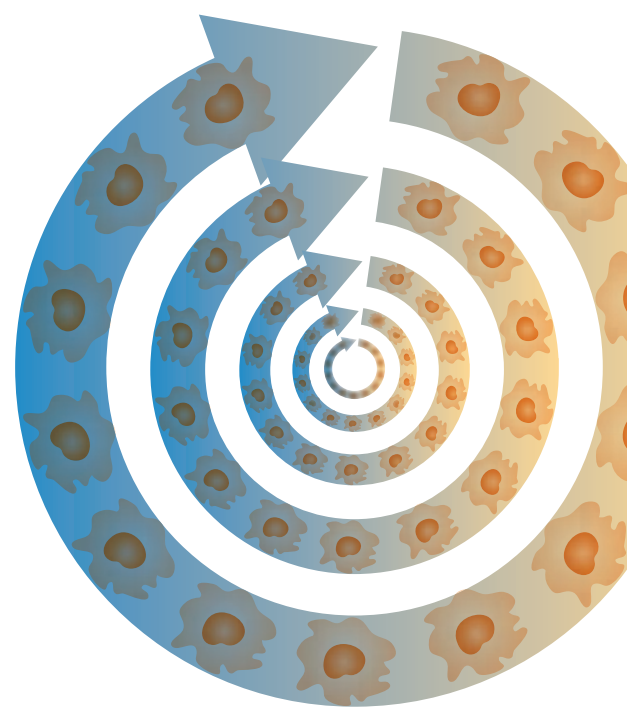

tissue macrophages do not. M-CSF responsiveness and monocyte differentiation are regulated by the transcription factors MAFB and MAF (also known as c-MAF), so the authors investigated the effect of deletion of MAFB and MAF on these processes. $\mathrm{Mafb}^{-/-} \mathrm{Maf}^{-/-}$blood leukocytes were highly efficient at forming M-CSF-induced colonies, which surprisingly seemed to arise from mature monocytes rather than progenitors. Indeed, a high frequency of colonies developed from purified mature $\mathrm{Mafb}^{-/} \mathrm{Maf}^{-1-}$ monocytes $\left(\mathrm{MACl}^{+} \mathrm{F} 4 / 80^{+} \mathrm{CD} 115^{+} \mathrm{KIT}^{-}\right)$, and they could even be serially re-plated and cultured continuously for 8 months without spoiling.

Remarkably, the high cloning and re-cloning efficiency of $\mathrm{Mafb}^{-/-} \mathrm{Maf}^{-/-}$ blood monocytes was not associated with de-differentiation, as the proliferating cells maintained differentiated functions and phenotype.

Neither was it associated with cellu-

lar transformation. Mice receiving in vitro expanded $\mathrm{Mafb}^{-/-} \mathrm{Maf}^{-/-}$ macrophages showed no signs of tumours, leukaemia or myeloproliferative disease for more than 6 months after transfer, despite the ability of these cells to proliferate in vivo. Instead, the transferred cells became integrated with the normal macrophage populations of the bone marrow, peritoneum, spleen and liver in the chimeric animals. Transplanted
$\mathrm{Mafb}^{-/-} \mathrm{Maf}^{-/-}$macrophages in the spleen and liver could participate in a characteristic host response to bacterial infection, showing a normal ability to phagocytose bacteria, localize to infected foci and express inducible nitric oxide synthase.

To understand how the selfrenewal of $\mathrm{Mafb}^{-1-} \mathrm{Maf}^{-1-}$ macrophages might be regulated, the authors looked at the transcription factors KLF4 and MYC, which are two of the four factors known to be sufficient to reprogramme differentiated cells into pluripotent stem cells. Consistent with a role in self-renewal capacity, $\mathrm{Mafb}^{-/-} \mathrm{Maf}^{-/-}$macrophages showed upregulated expression of both KLF4 and MYC compared with control cells. Moreover, knockdown of KLF4 and MYC expression using RNA interference significantly decreased the cloning and re-cloning efficiency of $\mathrm{Mafb}^{-/-} \mathrm{Maf}^{-/-}$cells.

So, the authors propose that loss of MAF proteins allows KLF4 and MYC to exert their self-renewal functions while being compatible with terminal differentiation. The ability to amplify functional differentiated cells without malignant transformation or stem cell intermediates could be an attractive prospect for cell-based therapies.

Lucy Bird

ORIGINAL RESEARCH PAPER Aziz, A., Soucie, E., Sarrazin, S. \& Sieweke, M. H. MafB/c-Maf deficiency enables self-renewal of differentiated functional macrophages. Science 326, 867-871 (2009) 